\title{
Increase in Diagnoses of Early Infectious Syphilis: Local Outbreak or Following the National Trend?

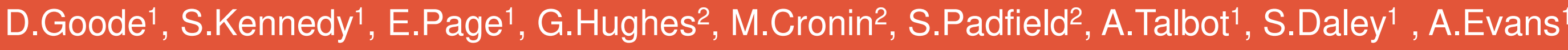

1. Department of Genitourinary Medicine, Leeds Teaching Hospitals/Leeds Sexual Health, Leeds. 2. Field Epidemiology Service, PHE, Yorkshire and Humber

\section{Background}

Increased incidence of early infectious syphilis (EIS) has been apparent in Leeds since sexual health services integrated in July 2015. National reports had previously shown a similar trend (1).

Concerns about a link between risk behaviour and the observed increase prompted a case note review to assess the demography and risk factors of this patient group and to identify links between syphilis cases.

\section{Results}

The database search for primary, secondary and early latent syphilis identified 76 cases in 2015 and 114 cases in 2016. This corresponded to an increase of $50 \%$ in comparison to a decrease of $5 \%$ in gonorrhoea cases.

Of the 76 cases recorded in 2015, one was excluded as it was miscoded and another was excluded as it was a duplicated code. In 2016114 cases were identified, one of which was excluded as the code was duplicated. In the 1st quarter of 2017, 35 cases were found; one case was excluded as the data could not be accessed. A total of 222 case notes were reviewed.

Interrupted time series analysis suggests that a significant change in the trend of syphilis diagnoses in Leeds occurred in July 2014 (Figure 1). Prior to this time point there was no significant change in monthly syphilis cases (Jan '12-Jul '14), but following July 2014 cases increased at a rate of 1.05 per month (95\% confidence interval 1.02 1.08; $P<0.001)$

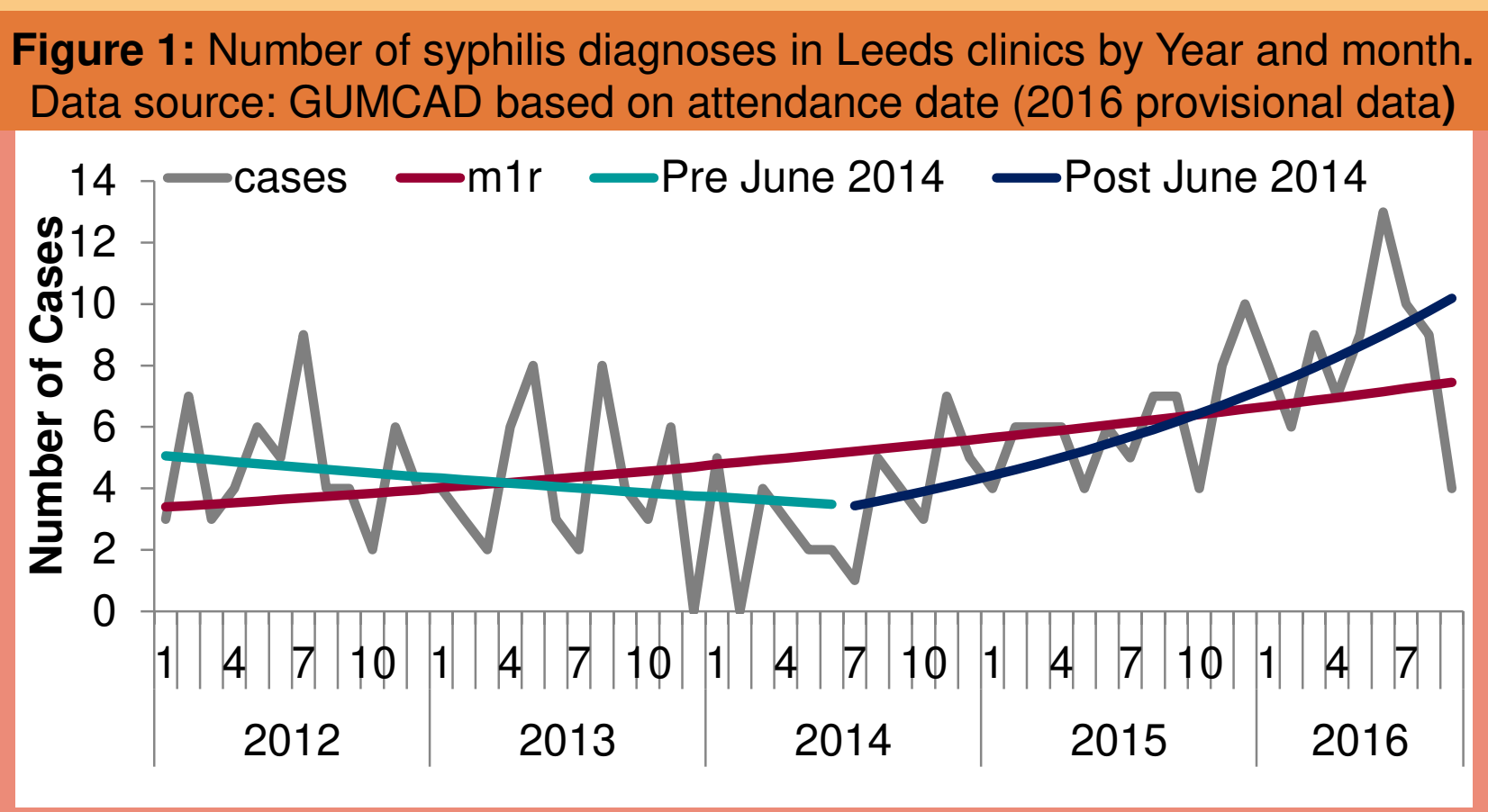

Syphilis cases were predominantly White British (79\%) and MSM (84\%). The highest incidence by age group was between 25 and 34 years (range 16-68) (Figure 2)

Overall, from 2015 to the first quarter $2017,75 \%$ of cases were $1^{\text {st }}$ infections, with $25 \%$ reporting previous treatment.

$74 \%$ of patients had a Leeds postcode and $2 \%$ were from outside of Yorkshire.

We were unable to establish whether there was any significant overlap in risk behaviour (eg saunas, chemsex parties, PrEP use) as absence was not consistently documented. After the identification of a

\section{Discussion}

Service reconfiguration in Leeds, focussed on MSM risk groups, coincided with increased EIS diagnosed within 1 year. However, epidemiological analyses indicate a sustained increase in syphilis diagnoses since 2014 , consistent with the national trend.

A single source of an outbreak has not been identified. Data would suggest the epidemiology of syphilis in Leeds is the similar to that reported in the UK as a whole. Following meetings with public health colleagues and our third sector partners, increasing diagnosis and raising awareness via an advertising campaign on dating applications and websites has been at the forefront of our plan to manage the spread of syphilis.

A particular concern has been that changes to sexual health services in Yorkshire may have resulted in an increase in out of area patients being treated in Leeds.

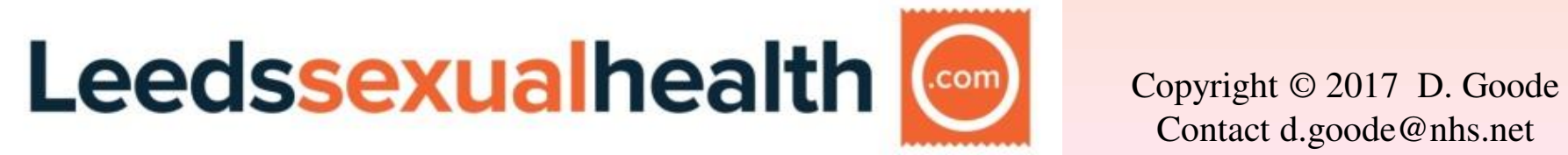

\section{Methods}

A retrospective case note review was performed on electronic patient records since the integration of sexual health services.

Case notes were assessed using BASHH audit standards, as well as being reviewed for several lifestyle risk factors. Interrupted time series analysis (Poisson regression) was used to assess whether the recent increase in syphilis diagnoses was associated with a significant change in trend at a particular point in time (a breakpoint) specified a priori based on visual inspection of the data.

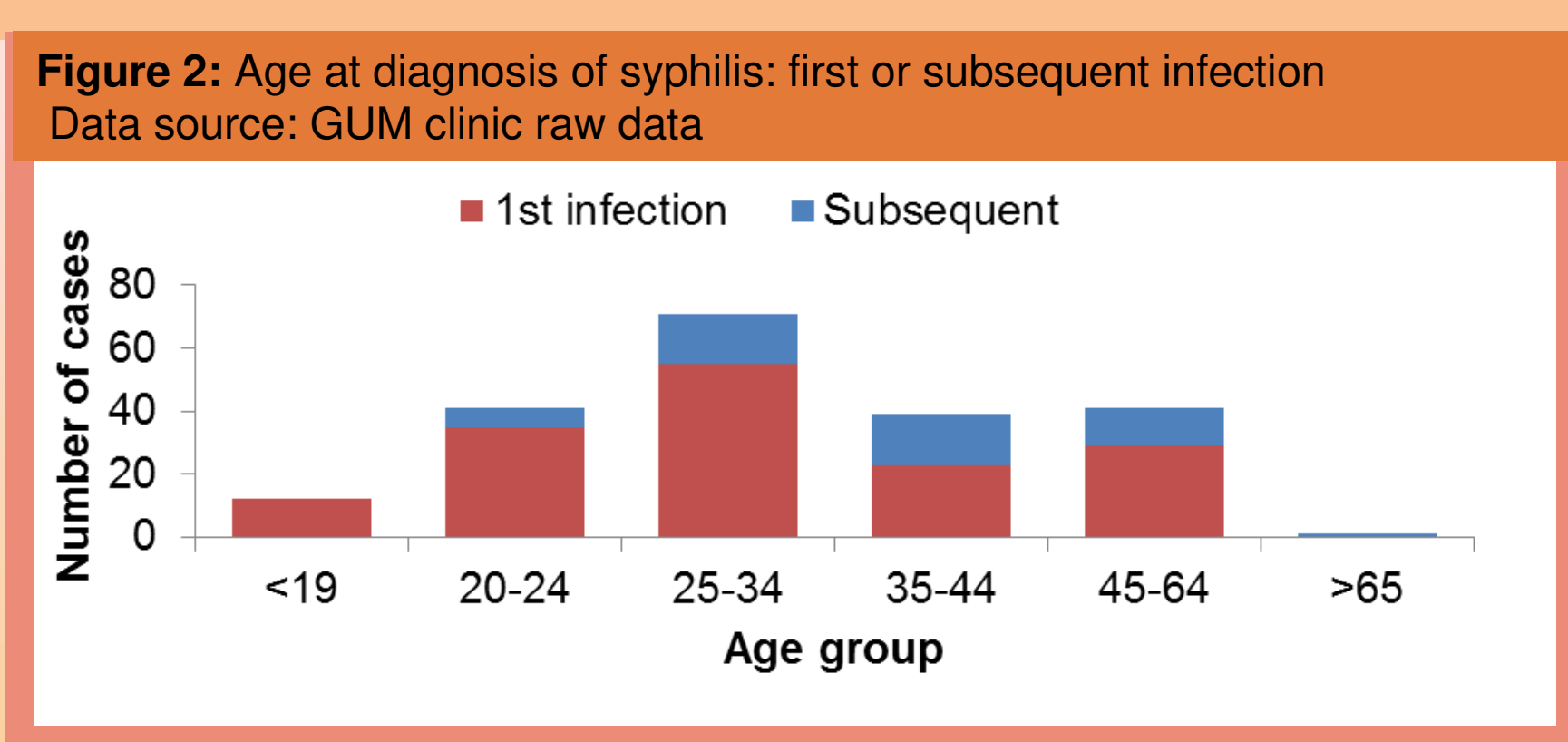

rise in diagnoses our syphilis proforma was changed to include compulsory questions on risk behaviour. This has resulted in an increased pick up of high risk behaviour from $11 \%$ of patient records (Jul-Dec 2015) to 42\% (Oct '16-Mar '17).

In the heterosexual cohort the most common risk factor was having sex with a partner from outside the UK.

The commonest co-infections in MSM with EIS were HIV (60\%), gonorrhoea then chlamydia, showing reciprocal age trends (figure 3 ).

Figure 3: Co infection at time of syphilis diagnosis in MSM by age and total heterosexual patients. Data source: GUM clinic raw data

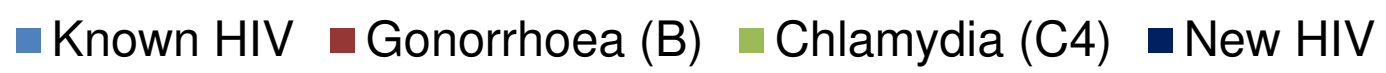

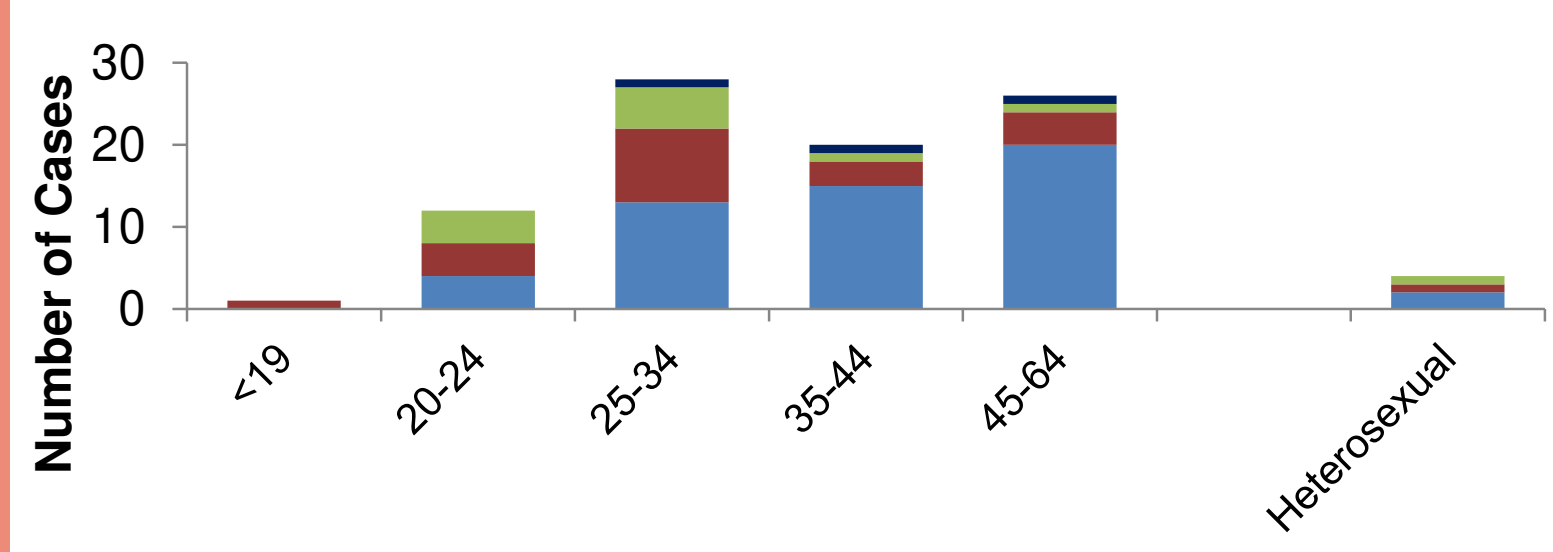

Eight patients had a syphilis re-infection during the look back period. Patients who required re-treatment due to non-abstinence were excluded. Of those 8 patients with syphilis re-infection, 3 had a primary syphilis diagnosis initially, but 7 had symptoms of primary syphilis at the time of their second diagnosis; 7 were MSM, 7 were white British and half were HIV positive. Four of the 8 re-infected patients had documented sauna or sex party attendance, chem-sex use, dating/hook up social media applications, or multiple city contacts as risk factors.

Most patients lived within 20 miles of Leeds Sexual Health services and in areas that were commuter areas for work in Leeds.

To address the need for increased case-finding, an evaluation of syphilis point of care testing (POCT), alongside capillary blood sampling, has been piloted at Leeds Sexual Health. The aim is to increase the opportunities for screening, particularly in outreach settings. The trial of these POCTs was started after the data from 2016 showed that $80 \%$ of these patients had a new infection. These are reported to have reduced sensitivity in EIS and are not useful in patients with previous history of syphilis. Of 15 used within clinic, 8 were positive and 7 of those were found to have EIS, the other had previous been treated. One of the 7 negative POCT was found to have EIS on serology. Internal evaluation continues. 